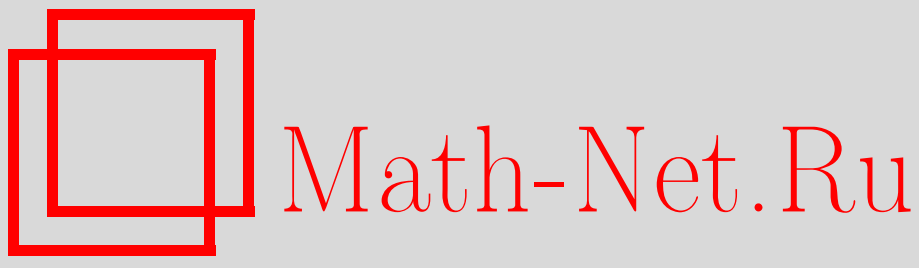

И. Э. Ниёзов, Регуляризация нестандартной задачи Коши для динамической системы Ламе, Изв. вузов. Матем., 2020, номер 4, 54-63

DOI: https://doi.org/10.26907/0021-3446-2020-4-54-63

Использование Общероссийского математического портала Math-Net.Ru подразумевает, что вы прочитали и согласны с пользовательским соглашением

http://www . mathnet.ru/rus/agreement

Параметры загрузки:

IP: 3.85 .73 .92

26 апреля 2023 г., 02:15:14

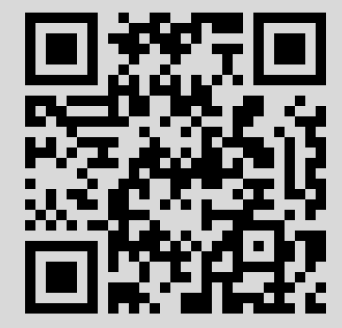


Известия вузов. Математика

2020, № 4, c. 54-63 https://kpfu.ru/science/nauchnye-izdaniya/ivrm

e-mail: izvuz.matem@kpfu.ru

\title{
И.Э. НИЁЗОВ
}

\section{РЕГУЛЯРИЗАЦИЯ НЕСТАНДАРТНОЙ ЗАДАЧИ КОШИ ДЛЯ ДИНАМИЧЕСКОЙ СИСТЕМЫ ЛАМЕ}

\begin{abstract}
Аннотация. Рассматривается задача Коши для динамических систем Ламе в цилиндре $G_{T}=$ $D \times(0, T)$, построенном над областью $D$ в трехмерном пространстве, с начальными данными, заданными на некоторой полосе на боковой поверхности цилиндра. Полоса имеет вид $S \times(0, T)$, где $S$ - открытое подмножество границы области $D$. Эта задача некорректная. При некоторых ограничениях на конфигурацию $S$, получена явная формула, описывающая решения этой задачи.
\end{abstract}

Ключевые слова: система Ламе, формула Карлемана, задача Коши, преобразование Лапласа.

УДК: 517.946

DOI: $10.26907 / 0021-3446-2020-4-54-63$

\section{ВВЕДЕНИЕ}

Наш подход к задаче Коши для параболических систем уравнений в цилиндре $G_{T}=$ $D \times(0, T)$, построенном над областью $D \in R^{3}$, с неполными граничными и начальными данными берет свое начало в работе [1]. Точнее, если применить преобразование Фурье или Лапласа по временной переменной $t \geq 0$ к параболическому уравнению, то это уравнение преобразуется в семейство эллиптических уравнений с комплексным параметром $\tau$ в области $D$. Аналогичные преобразования начальных данных на боковой поверхности цилиндра $G_{T}$ дают семейство задач Коши для эллиптического уравнения с параметром в области $D$ и начальными данными на некотором непустом открытом подмножестве $S$ границы $\partial D$. Эту задачу можно сразу решать конструктивными методами, тщательно разработанными в работе [2]. Затем остается применить обратное преобразование Лапласа.

Преобразование Фурье является важным методом изучения задач математической физики и дифференциальных уравнений. В классических работах предполагалось, что преобразования Фурье применяются к функциям, интегрируемым на бесконечности. В физике преобразования Фурье часто применяются к медленно растущим функциям без каких-либо достаточных обоснований. Строгая математическая теория преобразования Фурье для медленно растущих функций была впервые строго обоснована в работе [3]. Никакого систематического изучения преобразования Фурье для быстро растущих функций таких, как $e^{x}$, $e^{x^{2}}$ и т. д., не проводилось до появления работы [4], где была тщательно разработана теория преобразования Фурье для функций с произвольно быстрым ростом. Обобщение преобразования Фурье на случай быстро растущих функций имеет глубокую мотивацию. Напомним один из примеров, где для уравнения теплопроводности $u_{t}^{\prime}=u_{x x}^{\prime \prime}$ естественной областью, в которой задача Коши разрешима и имеет единственное решение, служит совокупность всех

Поступила в редакцию 15.03.2019, после доработки 15.03.2019. Принята к публикации 19.06.2019. 
функций, растущих при $|x| \rightarrow \infty$ медленнее, чем $e^{x^{2}}$, при каждом фиксированном $t \geq 0$. Заметим, что отличительным признаком работы [4] является выход в комплексную область и рассмотрение функционалов на классах аналитических (обычно целых) функций.

Мы будем изучать применимость данного подхода к задаче Коши для уравнения теплопроводности в цилиндре, построенном над областью типа "шапочки" $D$ в пространстве $R^{3}$. Применив преобразование Лапласа, получим задачу Коши для уравнения Гельмгольца в области $D$ с начальными данными на части границы этой области, лежащей в плоскости $x_{3}=0$. Потенциал уравнения Гельмгольца равен $i \tau$. Явная функция Карлемана была впервые получена в работе [5]. В работе [6] содержится частный случай построения функции Карлемана для задачи Коши для уравнения Гельмгольца в трехмерной области типа "шапочки".

\section{1. ЗАДАЧА КОШИ ДЛЯ ПАРАБОЛИЧЕСКИХ УРАВНЕНИЙ}

Пусть $D$ - ограниченная область с гладкой границей в $R^{3}$, и пусть $S$ - непустая открытая часть граничной поверхности $\partial D$. Рассмотрим задачу Коши для динамических систем уравнений Ламе в цилиндре $G_{T}=D \times(0, T)$ с начальными данными на полосе $S \times(0, T)$ боковой поверхности цилиндра, где $T>0$ - некоторое фиксированное число. Точнее, при заданных функциях $f$ на $G_{T}$ и $u_{0}, u_{1}$ на $S \times(0, T)$ требуется найти функцию $u \in C^{1}\left(\bar{G}_{T}\right) \cap C^{2}\left(G_{T}\right)$ в цилиндре $G_{T}$, удовлетворяющую условиям задачи

$$
\begin{aligned}
& \frac{\partial}{\partial t} u(x, t)=A\left(\partial_{x}\right) u(x, t)+f(x, t), \quad(x, t) \in G_{T} ; \\
& u(y, t)=u_{0}(y, t), \quad(y, t) \in S \times(0, T) ; \\
& T\left(\partial_{y}, n(y)\right) u(y, t)=u_{1}(y, t), \quad(y, t) \in S \times(0, T),
\end{aligned}
$$

где $A\left(\partial_{x}\right)=\mu \Delta+(\lambda+\mu) \operatorname{grad} \operatorname{div}, \mu>0,3 \lambda+2 \mu>0, \lambda, \mu$ - постоянные Ламе, $n(y)-$ единичный вектор внешней нормали к границе в точке $y \in \partial D$.

Из результатов работы [7] следует, что задача (1) имеет не более одного гладкого решения $u$.

На боковой поверхности нет характеристических точек динамических систем уравнений теории упругости. По теореме Коши-Ковалевской, если $S$ является действительно аналитической, то для всех действительных аналитических начальных данных $f$ и $u_{0}, u_{1}$ эта задача имеет локально близкое к $S \times(0, T)$ действительное аналитическое решение $u$. Из предыдущих соображений следует, что это решение единственно.

Чтобы построить приближенное решение задачи Коши (1), продолжим начальные данные $f$ и $u_{0}, u_{1}$ на весь полуцилиндр $t>0$, т. е. в дальнейшем будем считать $T=\infty$.

Теперь мы можем получить решение нестационарной задачи (1). Метод решения опирается на тот факт, что задачу (1) можно свести к некоторой другой задаче $(2)$, содержащей на одну независимую переменную меньше. Обе эти задачи эквивалентны, что устанавливается с помощью прямого и обратного преобразования Лапласа, но во многих случаях вторую задачу можно решить просто и в явной форме. Желательно, чтобы предположения, при которых можно применять преобразование, были достаточно широкими и охватили бы важные практические применения.

Мы потребуем, чтобы решение $u(x, t)$ задачи (1) удовлетворяло следующему условию: существует такое действительное $\alpha_{0}$, что функции

$$
u(x, t) e^{-\alpha_{0} t}, \quad \frac{\partial}{\partial x_{k}} u(x, t) e^{-\alpha_{0} t}, \quad \frac{\partial^{2}}{\partial x_{k} \partial x_{j}} u(x, t) e^{-\alpha_{0} t}
$$

остаются ограниченными равномерно по $x$ при стремлении $t$ к бесконечности. При этом предположении соответствующее преобразование Лапласа запишем в виде 


$$
U(x, \tau)=\mathcal{L} u(x, t)=\int_{0}^{\infty} u(x, t) e^{-\tau t} d t
$$

которое существует для $\operatorname{Re} \tau=\alpha>\alpha_{0}$ и является регулярной аналитической функцией от $\tau=\alpha+i \beta$ в полуплоскости $\alpha>\alpha_{0}$. На основании наших предположений мы получаем следующие формулы для производных этой функции:

$$
\begin{gathered}
\frac{\partial}{\partial x_{k}} U(x, \tau)=\mathcal{L}\left(\frac{\partial}{\partial x_{k}} u(x, t)\right)=\int_{0}^{\infty} \frac{\partial}{\partial x_{k}} u(x, t) e^{-\tau t} d t \\
\frac{\partial^{2}}{\partial x_{k} \partial x_{j}} U(x, \tau)=\mathcal{L}\left(\frac{\partial^{2}}{\partial x_{k} \partial x_{j}} u(x, t)\right)=\int_{0}^{\infty} \frac{\partial^{2}}{\partial x_{k} \partial x_{j}} u(x, t) e^{-\tau t} d t .
\end{gathered}
$$

Из наших условий заключаем, что преобразования Лапласа для функции $u_{t}(x, t)$ также существуют:

$$
\int_{0}^{T} u_{t}(x, t) e^{-\tau t} d t=\int_{0}^{T} e^{-\tau t} d u(x, t)=u(x, T) e^{-\tau T}-u(x, 0)+\tau \int_{0}^{T} u(x, t) e^{-\tau t} d t .
$$

Так как правая часть имеет предел при $T \rightarrow \infty$ и $\operatorname{Re} \tau>\alpha_{0}$, то левая часть также имеет предел, т. е.

$$
\int_{0}^{\infty} u_{t}(x, t) e^{-\tau t} d t=\tau U(x, \tau)-u(x, 0)
$$

Заметим, что значения $u(x, 0)$ на дне цилиндра однозначно определяются значениями $u(x, t)$ при малых $t>0$ при условии, что $u \in C^{1}\left(\overline{G_{T}}\right) \cap C^{2}\left(G_{T}\right)$. Следовательно, значения $u(x, 0)$ однозначно определяются значениями функций $u_{0}(y, t)$ и $u_{1}(y, t)$ на $S \times(0, T)$. Кроме того, если мы умножим уравнения системы (1) и данные Коши на $e^{-\tau t}$ и проинтегрируем их по $t$ от 0 до $\infty$, то получим семейство задач Коши

$$
\begin{aligned}
& A\left(\partial_{x}, \tau\right) U(x, \tau)=u(x, 0)-\mathcal{L} f(x, t)=\mathcal{F}(x, \tau), x \in D \\
& U(y, \tau)=U_{0}(y, \tau), y \in S \\
& T\left(\partial_{y}, n(y)\right) U(y, \tau)=U_{1}(y, \tau), y \in S
\end{aligned}
$$

где $A\left(\partial_{x}, \tau\right)=A\left(\partial_{x}\right)-\tau, U(y, \tau)=\mathcal{L} u(y, t), U_{0}(y, \tau)=\mathcal{L} u_{0}(x, t), U_{1}(y, \tau)=\mathcal{L} u_{1}(y, t)$ в области $D$, параметризованных комплексным параметром $\tau$, меняющимся вдоль вертикальной линии в $\operatorname{Re} \tau>\alpha_{0}$ полуплоскости. Для любого фиксированного $\tau$ соотношения (2) определяют задачу Коши для систем уравнений колебания, точнее для уравнения упруго-колебательного состояния среды $D$, т. е. для уравнения

$$
\mu \Delta U+(\lambda+\mu) \operatorname{grad} \operatorname{div} U-\tau U-\mathcal{F}=0
$$

в $D$ с начальными данными на части $S$. Хорошо известно [8]-[10], что эта задача неустойчива в естественной постановке, если $S$ не совпадает со всей границей, следовательно, что задача (1) некорректна.

В дальнейшем для решения задачи (2) используем метод, основанный на функции Карлемана [11], [12]. Матрицу фундаментальных решений однородного уравнения колебания

$$
\mu \Delta U+(\lambda+\mu) \operatorname{grad} \operatorname{div} U-\tau U=0
$$

представим в виде

$$
\begin{gathered}
\Psi(x, \tau)=\left\|\Psi_{k j}(x, \tau)\right\|_{3 \times 3} \\
\Psi_{k j}(x, \tau)=\sum_{q=1}^{2}\left(\delta_{k j} a_{q}+b_{q} \frac{\partial^{2}}{\partial x_{k} \partial x_{j}}\right)\left(\frac{\exp \left(i k_{q}|x|\right)}{|x|}\right),
\end{gathered}
$$


где

$$
k_{1}^{2}=\frac{-\tau}{\lambda+2 \mu}, \quad k_{2}^{2}=\frac{-\tau}{\mu}, \quad a_{q}=\frac{\delta_{2 q}}{2 \pi \mu}, \quad b_{q}=\frac{(-1)^{q-1}}{2 \pi \tau} .
$$

Верна формула Сомилиана-Бетти [13].

Теорема 1. Регулярное в $D$ решение $U(x, \tau)$ неоднородного уравнения колебания (3) представимо в виде

$$
\begin{array}{r}
2 U(x, \tau)=\int_{\partial D}\left(\Psi(y-x, \tau)\left\{T\left(\partial_{y}, n(y)\right) U(y, \tau)\right\}-\left\{T\left(\partial_{y}, n(y)\right) \Psi(y-x)\right\}^{*} U(y, \tau)\right) d s_{y}+ \\
+\int_{D} \Psi(y-x, \tau) \mathcal{F}(y, \tau) d y=\left\{\begin{array}{rr}
2 U(x, \tau), & x \in D \\
0, & x \notin \bar{D}
\end{array}\right.
\end{array}
$$

Пусть $D$ - ограниченная область в верхнем полупространстве $x_{3}>0$ пространства $R^{3}$, граница которого состоит из гладкой поверхности $S$, лежащей в полупространстве $x_{3}>0$, и замкнутых кусков плоскости $x_{3}=0$. Такие области обычно называются областями типа "шапочки". Заметим, что единичный вектор внешней нормали на части $\partial D \backslash S$ равен $(0,0,-1)$.

Пусть $x=\left(x_{1}, x_{2}, x_{3}\right)=\left(x^{\prime}, x_{3}\right)$ и $y=\left(y_{1}, y_{2}, y_{3}\right)=\left(y^{\prime}, y_{3}\right)$ - точки евклидового пространства $R^{3}$. Положим $\rho=\left|x^{\prime}-y^{\prime}\right|$ и определим функцию

$$
\Phi(y, x, \sigma, \Lambda)=\frac{1}{-2 \pi^{2} K\left(x_{3}\right)} \int_{0}^{\infty} \operatorname{Im} \frac{K(w)}{w-x_{3}} \frac{\cos (\Lambda u) d u}{\sqrt{u^{2}+\rho^{2}}}
$$

где $w=i \sqrt{u^{2}+\rho^{2}}+y_{3}, \Lambda$ - комплексное число, $K(w)=\exp \left(\sigma w^{2}\right), \sigma-$ положительный числовой параметр.

Выделив мнимую часть, получим

$$
\Phi(y, x, \sigma, \Lambda)=\int_{0}^{\infty} \mathcal{K}(y, x, \sigma, u) \cos (\Lambda u) d u
$$

где

$$
\begin{aligned}
& \mathcal{K}(y, x, \sigma, u)=\frac{1}{-2 \pi^{2}} \frac{\exp \sigma\left(y_{3}^{2}-x_{3}^{2}\right) \exp \left(-\sigma\left(u^{2}+\rho^{2}\right)\right)}{u^{2}+r^{2}} \times \\
& \times\left(\frac{\left(y_{3}-x_{3}\right) \sin \left(2 \sigma y_{3} \sqrt{u^{2}+\rho^{2}}\right)}{\sqrt{u^{2}+\rho^{2}}}-\cos \left(2 \sigma y_{3} \sqrt{u^{2}+\rho^{2}}\right)\right) .
\end{aligned}
$$

Таким образом, сходимость несобственного интеграла в правой части соотношения (6) обеспечивается множителем $\exp \left(-\sigma u^{2}\right)$.

Лемма 1 ([10]). Функиия $\Phi(y, x, \sigma, \Lambda)$, определяемая формулой $(6)$, представима в виде

$$
\Phi(y, x, \sigma, \Lambda)=-\frac{1}{4 \pi} \frac{\exp (i \Lambda r)}{r}+g(y, x, \sigma, \Lambda),
$$

где $g(y, x, \sigma, \Lambda)$ - некоторая дваждъ нерерьвно дифферениируемая функиия по у, удовлетворяющая по у уравнению Гельмголвца: $\Delta_{y} g-\Lambda^{2} g=0$, включая и точку $y=x$. 
Функцию $\Phi(y, x, \sigma, \Lambda)$ назовем функцией Карлемана для уравнения Гельмгольца.

Определение. Матрицей Карлемана задачи (1.2) называется $3 \times 3$-матрица $\Pi(y, x, \sigma, \Lambda)$, зависящая от двух точек $x, y$ и положительного числового параметра $\sigma$, удовлетворяет следующим двум условиям:

1) $\Pi(y, x, \sigma, \Lambda)=\Psi(y-x, \tau)+G(y, x, \sigma, \tau)$, где матрица $G(y, x, \sigma, \tau)$ удовлетворяет по переменной $y$ уравнению (4) всюду в области $D, \Psi(y-x, \tau)$ - матрица фундаментальных решений системы $(2)$;

2) $\int_{\partial D \backslash S}\left(|\Pi(y, x, \sigma, \Lambda)|+\left|T\left(\partial_{y}, n(y)\right)\right|\right) d s_{y} \leq \varepsilon(\sigma)$, где $\varepsilon(\sigma) \rightarrow 0$ при $\sigma \rightarrow \infty ;|\Pi|-$ евклидова норма матрицы $\|\Pi\|$, т. е. $|\Pi|=\left(\sum_{k, j=1}^{3} \Pi_{k j}^{2}\right)^{1 / 2}$, в частности, $|U|=\left(\sum_{k=1}^{3} U_{k}^{2}\right)^{1 / 2}$.

Известно [4], что для регулярных $u, v$ и $A\left(\partial_{x}, \tau\right) u, A\left(\partial_{x}, \tau\right) v$, абсолютно интегрируемых в $D$ вектор-функций, верна формула

$$
\int_{D}\left[v A\left(\partial_{y}, \tau\right) u-u A\left(\partial_{y}, \tau\right) v\right] d y=\int_{\partial D}\left[v T\left(\partial_{y}, n(y)\right) u-u T\left(\partial_{y}, n(y)\right) v\right] d s_{y} .
$$

Подставляя в это равенство вместо $v=G(y, x, \sigma, \tau)$ и $u=U(x, \tau)$ регулярное решение системы (3), получим

$$
\begin{aligned}
\int_{\partial D}\left(G(y, x, \sigma, \tau)\left\{T\left(\partial_{y}, n(y)\right) U(y, \tau)\right\}-\left\{T\left(\partial_{y}, n(y)\right)\right.\right. & \left.G(y, x, \sigma, \tau)\}^{*} U(y, \tau)\right) d s_{y}+ \\
& +\int_{D} G(y, x, \sigma, \tau) \mathcal{F}(y, \tau) d y=0 .
\end{aligned}
$$

Прибавляя (5) и (7), имеем

$$
\begin{aligned}
\int_{\partial D}((\Psi(y-x, \tau) & \\
& G(y, x, \sigma, \tau))\left\{T\left(\partial_{y}, n(y)\right) U(y, \tau)\right\}- \\
\left.-\left\{T\left(\partial_{y}, n(y)\right)(\Psi(y-x, \tau)+G(y, x, \sigma, \tau))\right\}^{*} U(y, \tau)\right) d s_{y}+ & \\
& +\int_{D}(\Psi(y-x, \tau)+G(y, x, \sigma, \tau)) \mathcal{F}(y, \tau) d y=\left\{\begin{array}{cc}
2 U(x, \tau), & x \in D ; \\
0, & x \notin \bar{D} .
\end{array}\right.
\end{aligned}
$$

Из (8) видно, что если матрица Карлемана задачи (2) известна, то верна

Теорема 2. Всякое регулярное решение $U(x, \tau)$ системы (3) в области D определяется формулой

$$
\begin{aligned}
\int_{\partial D}\left(\Pi(y, x, \sigma, \tau)\left\{T\left(\partial_{y}, n(y)\right) U(y, \tau)\right\}-\left\{T\left(\partial_{y}, n(y)\right) \Pi(y, x, \sigma, \tau)\right\}^{*} U(y, \tau)\right) d s_{y}+ \\
+\int_{D} \Pi(y, x, \sigma, \tau) \mathcal{F}(y, \tau) d y=\left\{\begin{array}{cc}
2 U(x, \tau), & x \in D ; \\
0, & x \notin \bar{D}
\end{array}\right.
\end{aligned}
$$

где $(y, x, \sigma, \tau)$ - матрица Карлемана.

Используя матрицу Карлемана, легко вывести оценку устойчивости решения задачи Коши (2), а также указать метод эффективного решения этой задачи. 
С целью построения приближенного решения задачи (2) построим матрицу Карлемана

$$
\Pi(y, x, \sigma, \tau)=\left\|\Pi_{k j}(y, x, \sigma, \tau)\right\|_{3 \times 3},
$$

где

$$
\Pi_{k j}(y, x, \sigma, \tau)=\sum_{q=1}^{2}\left(\delta_{k j} a_{q}+b_{q} \frac{\partial^{2}}{\partial x_{k} \partial x_{j}}\right) \Phi\left(y, x, \sigma, i k_{q}\right),
$$

$\Phi\left(y, x, \sigma, i k_{q}\right)$ из $(6)$.

Лемма 2. Матрица $\Pi_{k j}(y, x, \sigma, \tau)$, заданная формулой $(10)$, является матрицей Карлемана задачи (2).

Доказательство леммы вытекает из леммы 1 и (10).

\section{2. РЕШЕНИЕ ЗАДАЧИ КОШИ (2)}

Обозначим

$$
\begin{aligned}
2 U_{\sigma}(x, \tau)=\int_{S}( & \Pi(y, x, \sigma, \tau)\left\{T\left(\partial_{y}, n(y)\right) U(y, \tau)\right\}- \\
& \left.\quad-\left\{T\left(\partial_{y}, n(y)\right) \Pi(y, x, \sigma, \tau)\right\}^{*} U(y, \tau)\right) d s_{y}+\int_{D} \Pi(y, x, \sigma, \tau) \mathcal{F}(y, \tau) d y
\end{aligned}
$$

Теорема 3. Пусть $U(x, \tau)$ - регулярное решение уравнение (3) в области $D$, удовлетворяющее условию

$$
|U(y, \tau)|+\left|T\left(\partial_{y}, n(y)\right) U(y, \tau)\right| \leq M, \quad y \in \partial D,
$$

тогда при $\sigma \geq 1$ справедлива оченка

$$
\left|U(y, \tau)-U_{\sigma}(x, \tau)\right| \leq M C(\lambda, \mu, \tau, x) \sigma^{2} \exp \left(-\sigma x_{3}^{2}\right), \quad y \in D,
$$

¿де

$$
C(\lambda, \mu, \tau, x)=C(\lambda, \mu, \tau) \int_{\partial D} \frac{d s_{y}}{r^{2}} .
$$

Доказательство. Рассмотрим разность

$$
\begin{gathered}
\left|U(x, \tau)-U_{\sigma}(x, \tau)\right| \leq \frac{1}{2} \mid \int_{\partial D \backslash S}\left(\Pi(y, x, \sigma, \tau)\left\{T\left(\partial_{y}, n(y)\right) U(y, \tau)\right\}-\right. \\
\left.-\left\{T\left(\partial_{y}, n(y)\right) \Pi(y, x, \sigma, \tau)\right\}^{*} U(y, \tau)\right) d s_{y} \mid \leq \frac{1}{2} \int_{\partial D \backslash S}\left[|\Pi(y, x, \sigma, \tau)|+\left|T\left(\partial_{y}, n(y)\right) \Pi(y, x, \sigma, \tau)\right|\right] \times \\
\times\left[|U(y, \tau)|+\left|T\left(\partial_{y}, n(y)\right) U(y, \tau)\right|\right] d s_{y} \leq \frac{M}{2} \int_{\partial D \backslash S}\left[|\Pi(y, x, \sigma, \tau)|+\left|T\left(\partial_{y}, n(y)\right) \Pi(y, x, \sigma, \tau)\right|\right] .
\end{gathered}
$$

С учетом (6), (10)

$$
\begin{gathered}
\left|\Phi\left(y, x, \sigma, i k_{q}\right)\right| \leq C_{1} r^{-1} \exp \sigma\left(y_{3}^{2}-x_{3}^{2}\right), \\
\left|\frac{\partial \Phi\left(y, x, \sigma, i k_{q}\right)}{\partial y_{k}}\right| \leq C_{2} r^{-2} \sigma \exp \sigma\left(y_{3}^{2}-x_{3}^{2}\right), \\
\left|\frac{\partial^{2} \Phi\left(y, x, \sigma, i k_{q}\right)}{\partial y_{k} \partial y_{j}}\right| \leq C_{3} r^{-3} \sigma^{2} \exp \sigma\left(y_{3}^{2}-x_{3}^{2}\right) .
\end{gathered}
$$


Соответственно

$$
|\Pi(y, x, \sigma, \tau)|+\left|T\left(\partial_{y}, n(y)\right) \Pi(y, x, \sigma, \tau)\right| \leq C(\lambda, \mu, \tau) r^{-3} \sigma^{2} \exp \sigma\left(y_{3}^{2}-x_{3}^{2}\right) .
$$

Как видим, матрица П $(y, x, \sigma, \tau)$ является фундаментальным решением типа свертки для оператора $A\left(\partial_{x}, \tau\right)$ в $R^{3}$. Тогда для фиксированного $\tau$ приближенное решение задачи (2) получается из так называемой формулы Карлемана

$$
\begin{gathered}
U(x, \tau)=\lim _{\sigma \rightarrow \infty} U_{\sigma}(x, \tau)=\frac{1}{2} \lim _{\sigma \rightarrow \infty}\left(\int_{S} \Pi(y, x, \sigma, \tau)\left\{T\left(\partial_{y}, n(y)\right) U(y, \tau)\right\} d s_{y}-\right. \\
-\int_{S}\left\{T\left(\partial_{y}, n(y)\right) \Pi(y, x, \sigma, \tau)\right\}^{*} U(y, \tau) d s_{y}+ \\
\left.+\int_{D} \Pi(y, x, \sigma, \tau)(\mu \Delta+(\lambda+\mu) \operatorname{graddiv}-\tau) U(y, \tau) d y\right), \quad x \in D .
\end{gathered}
$$

Предположим, что обратное преобразование Лапласа возможно при переходе к пределу в последней формуле:

$$
\begin{gathered}
\mathcal{L}^{-1} U(x, \tau)=\lim _{\sigma \rightarrow \infty} \mathcal{L}^{-1} U_{\sigma}(x, \tau)=\frac{1}{2} \lim _{\sigma \rightarrow \infty}\left(\int_{S} \mathcal{L}^{-1}\left[\Pi(y, x, \sigma, \tau)\left\{T\left(\partial_{y}, n(y)\right) U(y, \tau)\right\}\right] d s_{y}-\right. \\
\quad-\int_{S} \mathcal{L}^{-1}\left[\left\{T\left(\partial_{y}, n(y)\right) \Pi(y, x, \sigma, \tau)\right\}^{*} U(y, \tau)\right] d s_{y}+ \\
\left.+\int_{D} \mathcal{L}^{-1}[\Pi(y, x, \sigma, \tau)(\mu \Delta+(\lambda+\mu) \text { graddiv }-\tau) U(y, \tau)] d y\right), x \in D .
\end{gathered}
$$

В силу известной теоремы о свертке для преобразования Лапласа получим

$$
\begin{aligned}
\mathcal{L}^{-1}\left[\Pi(y, x, \sigma, \tau)\left\{T\left(\partial_{y}, n(y)\right) U(y, \tau)\right\}\right]=\mathcal{L}^{-1}[\Pi(y, x, \sigma, \tau)] *\left\{T\left(\partial_{y}, n(y)\right) \mathcal{L}^{-1} U(y, \tau)\right\}= \\
=\int_{0}^{t}\left[\left(\mathcal{L}^{-1} \Pi\right)\left(y, x, \sigma, t-t^{\prime}\right)\right]\left\{T\left(\partial_{y}, n(y)\right) u\left(y, t^{\prime}\right)\right\} d t^{\prime} .
\end{aligned}
$$

Аналогично,

$$
\mathcal{L}^{-1}\left[\left\{T\left(\partial_{y}, n(y)\right) \Pi(y, x, \sigma, \tau)\right\}^{*} U(y, \tau)\right]=\int_{0}^{t}\left\{T\left(\partial_{y}, n(y)\right)\left(\mathcal{L}^{-1} \Pi\right)\left(y, x, \sigma, t-t^{\prime}\right)\right\} u\left(y, t^{\prime}\right) d t^{\prime}
$$

и

$$
\begin{gathered}
\mathcal{L}^{-1}[\Pi(y, x, \sigma, \tau)(\mu \Delta+(\lambda+\mu) \text { graddiv }-\tau) U(y, \tau)]= \\
=\int_{0}^{t}\left[\left(\mathcal{L}^{-1} \Pi\right)\left(y, x, \sigma, t-t^{\prime}\right)\right]\left(\mu \Delta+(\lambda+\mu) \text { graddiv }-\frac{\partial}{\partial t^{\prime}}\right) u\left(y, t^{\prime}\right) d t^{\prime}- \\
-\left(\mathcal{L}^{-1} \Pi\right)(y, x, \sigma, t) u(x, 0) .
\end{gathered}
$$


Следовательно, применяя обратное преобразование Лапласа, получим формулу

$$
\begin{gathered}
u(x, t)=\frac{1}{2} \lim _{\sigma \rightarrow \infty}\left(-\int_{D}\left(\mathcal{L}^{-1} \Pi\right)(y, x, \sigma, t) u(x, 0) d y+\right. \\
+\int_{S} \int_{0}^{t}\left(\mathcal{L}^{-1} \Pi\right)\left(y, x, \sigma, t-t^{\prime}\right)\left\{T\left(\partial_{y}, n(y)\right) u\left(y, t^{\prime}\right)\right\} d s_{y} d t^{\prime}- \\
-\int_{S} \int_{0}^{t}\left\{T\left(\partial_{y}, n(y)\right)\left(\mathcal{L}^{-1} \Pi\right)\left(y, x, \sigma, t-t^{\prime}\right)\right\} u\left(y, t^{\prime}\right) d s_{y} d t^{\prime}+ \\
\left.+\int_{D} \int_{0}^{t}\left[\left(\mathcal{L}^{-1} \Pi\right)\left(y, x, \sigma, t-t^{\prime}\right)\right]\left(\mu \Delta+(\lambda+\mu) \operatorname{grad} \operatorname{div}-\frac{\partial}{\partial t^{\prime}}\right) u\left(y, t^{\prime}\right) d y d t^{\prime}\right)
\end{gathered}
$$

для любых $(x, t) \in G_{T}$.

Положим

$$
\mathcal{P}(y, x, \sigma, t)=\left(\mathcal{L}^{-1} \Pi\right)(y, x, \sigma, t)=\frac{1}{4 \pi} \int_{\Re(\tau)=\alpha} e^{\tau t} \Pi(y, x, \sigma, \tau) d \tau
$$

при $(x, y)$, лежащих вне диагонали в $D \times D$, и при $t>0$, где $\alpha-$ достаточно малое отрицательное число. Простые вычисления показывают, что

$$
\left(A\left(\partial_{y}\right)-\frac{\partial}{\partial t}\right) \mathcal{P}(y, x, \sigma, t)=\frac{1}{2 \pi i} \int_{\Re(\tau)=\alpha} e^{\tau t} A\left(\partial_{y}, \tau\right) \Pi(y, x, \sigma, \tau) d \tau=0,
$$

для $(x, y)$ и $t$, лежащих в области определения функции $\mathcal{P}$. Кроме того, $\mathcal{P}(y, x, \sigma, t)$ в некотором смысле стремится к нулю по $y$ при $\sigma \rightarrow \infty$ вне $S$ на границе области $D$.

Находим вид $\mathcal{P}(y, x, \sigma, t)$ для областей типа "шапочки". Для этого рассмотрим функцию $\Phi(y, x, \sigma, \Lambda)$ из (7) при $\Lambda=\sqrt{\tau}$

$$
\Phi(y, x, \sigma, \tau)=\int_{0}^{\infty} \mathcal{K}(y, x, \sigma, u) \cos (\sqrt{\tau} u) d u .
$$

На плоскости $y_{3}=0$

$$
\mathcal{K}(y, x, \sigma, u)=\frac{1}{2 \pi^{2}} \frac{\exp \left(-\sigma x_{3}^{2}\right) \exp \left(-\sigma\left(u^{2}+\rho^{2}\right)\right)}{u^{2}+r^{2}} .
$$

Пусть

$$
\mathbb{F}(y, x, \sigma, t)=\mathcal{L}^{-1} \Phi(y, x, \sigma, \tau)=\int_{0}^{\infty} \mathcal{K}(y, x, \sigma, u) \mathcal{L}^{-1}(\cos (\sqrt{\tau} u)) d u
$$

тогда

$$
\begin{gathered}
\mathcal{P}(y, x, \sigma, t)=\left(\mathcal{L}^{-1} \Pi\right)(y, x, \sigma, t), \\
\mathcal{P}(y, x, \sigma, t)=\left\|\mathcal{L}^{-1} \Pi_{k j}(y, x, \sigma, \tau)\right\|_{3 \times 3}=\left\|\mathcal{P}_{k j}(y, x, \sigma, t)\right\|_{3 \times 3},
\end{gathered}
$$


где

$$
\mathcal{P}_{k j}(y, x, \sigma, t)=\sum_{q=1}^{2}\left(\delta_{k j} a_{q}+b_{q} \frac{\partial^{2}}{\partial x_{k} \partial x_{j}}\right) \mathbb{F}(y, x, \sigma, t) .
$$

Лемма 3. Если $\mathcal{P}(y, x, \sigma, t)$ - матрица Карлемана задачи (1), то верна формула

$$
\begin{gathered}
u(x, t)=\frac{1}{2} \lim _{\sigma \rightarrow \infty}\left(-\int_{D}(\mathcal{P}(y, x, \sigma, t) u(x, 0) d y+\right. \\
+\int_{S} \int_{0}^{t} \mathcal{P}\left(y, x, \sigma, t-t^{\prime}\right)\left\{T\left(\partial_{y}, n(y)\right) u\left(y, t^{\prime}\right)\right\} d s_{y} d t^{\prime}- \\
-\int_{S} \int_{0}^{t}\left\{T\left(\partial_{y}, n(y)\right) \mathcal{P}\left(y, x, \sigma, t-t^{\prime}\right)\right\} u\left(y, t^{\prime}\right) d s_{y} d t^{\prime}+ \\
\left.+\int_{D} \int_{0}^{t} \mathcal{P}\left(y, x, \sigma, t-t^{\prime}\right)\left(\mu \Delta+(\lambda+\mu) \operatorname{grad} \operatorname{div}-\frac{\partial}{\partial t^{\prime}}\right) u\left(y, t^{\prime}\right) d y d t^{\prime}\right)
\end{gathered}
$$

дляв всех $(x, t) \in G_{T}$.

Как видно, что все последние выражения зависят от величины

$$
\mathcal{L}^{-1}(\cos (\sqrt{\tau} u))=\frac{1}{2 \pi i} \int_{\Re(p)=\alpha} e^{p t} \cos (\sqrt{p} u) d p=0 .
$$

Но функция $\cos (\sqrt{p})$ не является преобразованием Лапласа для функции на неотрицательной полуоси, удовлетворяющей условию $|f(t)| \leq C \exp (k t)$ при $t \geq 0$. Обратное преобразованием Лапласа для функции $\cos (\sqrt{p})$ следует интерпретировать в рамках более сложной теории, развитой в работе [4].

Теорема 4. Пусть $D$ - область типа "шапочки"в пространстве $R^{3}$. Тогда для любого $u \in H^{2,1}\left(G_{T}\right)$ имеем

$$
\begin{aligned}
u(x, t)=\frac{1}{2} \lim _{\sigma \rightarrow \infty}\left(-\int_{D} \mathcal{P}(y, x, \sigma, t) u(x, 0) d y+\int_{S} \int_{0}^{t} \mathcal{P}\left(y, x, \sigma, t-t^{\prime}\right)\left\{T\left(\partial_{y}, n(y)\right) u\left(y, t^{\prime}\right)\right\} d s_{y} d t^{\prime}-\right. \\
\quad-\int_{S} \int_{0}^{t}\left\{T\left(\partial_{y}, n(y)\right) \mathcal{P}\left(y, x, \sigma, t-t^{\prime}\right)\right\} u\left(y, t^{\prime}\right) d s_{y} d t^{\prime}+ \\
\left.+\int_{D} \int_{0}^{t} \mathcal{P}\left(y, x, \sigma, t-t^{\prime}\right)\left(\mu \Delta+(\lambda+\mu) \operatorname{grad} \operatorname{div}-\frac{\partial}{\partial t^{\prime}}\right) u\left(y, t^{\prime}\right) d y d t^{\prime}\right)
\end{aligned}
$$

для всех $(x, t) \in G_{T}$.

Доказательство утверждения теоремы непосредственно следует из леммы 3 и формулы (1.5). 


\section{Литература}

[1] Агранович М.С., Вишик М.И. Эллиптические задачи с параметром и параболические задачи общего вида, УМH, 19:3 (117), 53-161 (1964).

[2] Tarkhanov N.N. The Cauchy problem for solutions of elliptic equations, 7, Akad. Verl., Berlin, (1995).

[3] Bochner S. Vorlesungen über Fouriersche Integrale, Akad. Verl., Leipzig, (1932).

[4] Гельфанд И.М., Шилов Г.Е. Преобразования Фуръе быстро растущих функиий и вопросы единственности решения задачи Коши, УМН, 8:6 (58), 3-54 (1953).

[5] Ярмухамедов Ш. О задаче Коши для уравнение Лапласа, ДАН СССР 235 (2), 281-283 (1977).

[6] Yarmukhamedov Sh., Yarmukhamedov I. The Cauchy problem for the Helmholtz equation, Ill-Posed and Non-Classical Probl. Math. Physics and Anal., VSP, Utrecht, 143-172 (2003).

[7] Ландис Е.М., Олейник О.А. Обобщеннал аналитичность и некоторые связанные с ней свойства решений эллиптических и параболических уравнений, УМН, 29:2 (176), 190-206 (1974).

[8] Лаврентьев М.М., Романов В.Г., Шишатский С.П. Некорректнъе задачи математической физики и анализа (Наука, М., 1980).

[9] Puzyrev R.E., Shlapunov A.A. On an ill-posed problem for the heat equation, Журн. СФУ. Сер. Матем. и физ. 5 (3), 337-348 (2012).

[10] Махмудов К.О., Махмудов О.И., Тарханов Н.Н. Нестандартная задача Коши для уравнения теплопроводности, Матем. заметки 102 (2), 270-283 (2017).

[11] Ярмухамедов Ш. Функиия Карлемана и задача Коши для уравнения Лапласа, Сиб. матем. журн. 45 (3), 702-719 (2004)

[12] Makmudov O., Niyozov I., Tarkhanov N. The Cauchy problem of couple-stress elasticity, Complex anal. and dynamical systems III, Contemp. Math., 455, Amer. Math. Soc., Providence, RI, 297-310 (2008).

[13] Купрадзе В.Д., Гегелиа Т.Г., Башелейшвили М.О., Бурчуладзе Т.В. Трехмерные задачи математической теории упругости и термоупругости (Наука, М., 1976).

Икбол Эргашевич Ниёзов

Самаркандский государственный университет,

Университетский бульвар, д. 15, г. Самарканд, 140104, Узбекистан,

e-mail : iqboln@mail.ru

\section{I.E. Niyozov}

\section{Regularization of a nonstandard Cauchy problem for a dynamic Lame system}

Abstract. We consider a Cauchy problem for the dynamic systems Lame in a cylinder $G_{T}=$ $D \times(0, T)$ over a domain $D$ in $R^{3}$ with data on a strip lying on the lateral surface. The strip is of the form $S \times(0, T)$, where $S$ - is an open subset of the boundary of $D$. The problem is ill-posed. Under natural restrictions on the configuration of $S$ we derive an explicit formula for solutions of this problem.

Keywords: Cauchy problem, system theory of elasticity, elliptic system, ill-posed problem.

Ikbol Ergashevich Niyozov

Samarkand State University, 15 Universitetskii blvd., Samarkand, 140104 Republic of Uzbekistan,

e-mail: iqboln@mail.ru 